\title{
FLOW REGIMES FOR A MAGNETIC SUSPENSION UNDER A ROTATING MAGNETIC FIELD (*)
}

\author{
R. MAILFERT and A. MARTINET \\ Laboratoire de Physique des Solides (**) \\ Université de Paris-Sud, Centre d'Orsay, 91-Orsay (France)
}

(Reçu le 24 mars 1972)

\begin{abstract}
Résumé. - Nous avons effectué des observations qualitatives à des échelles macroscopique et locale sur les mouvements à l'intérieur d'un ferrofluide et d'une suspension de grains magnétiques, sous l'effet d'un champ magnétique tournant $\mathbf{H}$. Les grains individuels tournent de façon synchrone. Dans ces conditions, nous trouvons que :

- Quand le vecteur aimantation macroscopique $\mathbf{M}$ est uniforme sur tout l'échantillon, il n'y a pas de rotation d'ensemble du liquide.

De petits écarts à cette uniformité sont suffisants pour produire des rotations asynchrones du liquide, dans un sens ou dans l'autre, selon la distribution des pôles magnétiques à l'intérieur de l'échantillon.

- On peut très facilement créer de forts écarts à cette uniformité et, par suite, d'importantes rotations d'ensemble par l'intermédiaire de champs statiques locaux ou de concentrations de grains non uniformes.

Abstract. - We have performed qualitative observations of both macroscopic and local motions in a ferrofluid and in a suspension of magnetic grains under a rotating magnetic field $\mathbf{H}$. The individual grains rotate synchronously. We then find that :

- When the macroscopic magnetization vector $\mathbf{M}$ is uniform over the whole sample volume, there is no bulk rotation of the liquid.

- Slight departures from uniformity are enough to induce asynchronous rotations of the liquid of either sign, depending on the distribution of magnetic poles in the sample.

- Large departures from uniformity and powerful bulk rotations can be imposed very easily either by inhomogeneous static fields or grain concentrations.
\end{abstract}

1. Introduction. - Moskowitz and Rosensweig [1] have carried out some very interesting measurements on magnetic colloids driven by a rotating field $H_{\mathrm{r}}$. Typically, for $H_{\mathrm{r}}=40 \mathrm{Oe}$ and a rotation frequency of $100 \mathrm{~Hz}$, the fluid rotation was of the order of $1 \mathrm{~Hz}$. However, the theoretical analysis on which they based their interpretation is open to doubt, as has recently been shown in detail by Jenkins [2] : in fact, for a uniform distribution of parallel and non-interacting grains under a uniform field $H_{\mathrm{r}}$, there should be no macroscopic motion of the fluid. This can be seen from the magnetic poles model, shown in figure 1 . For a uniform magnetization, the magnetic poles appear on the outer boundary (Fig. 1c). Thus the force on the magnetic poles is applied at the solid/ liquid interface where the boundary conditions impose $v=0$ and no bulk rotation can take place.

(*) Work supported in part by Direction des Recherches et Moyens d'Essais.

(**) Laboratoire associé au CNRS
The same argument shows that bulk rotation is possible whenever the magnetic poles appear at some point inside the liquid (Fig. $1 a, 1 b$ ). We wish to report experimental observations which confirm this model and to consider in some detail a number of situations which lead to the appearance of magnetic poles in the liquid and consequently to bulk rotation : inhomo-

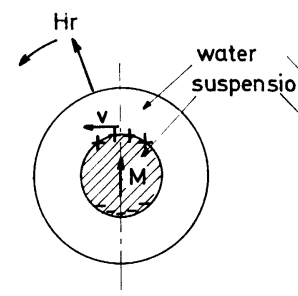

a)

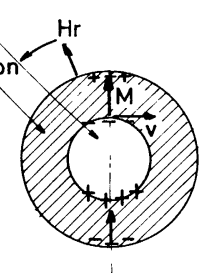

b)

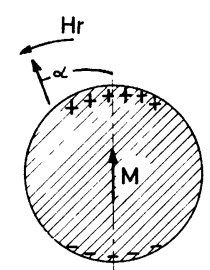

c)
FIG. 1. - Local forces on a magnetic colloid with magnetization $\mathrm{M}$ under a rotating field $H_{\mathrm{r}}$. The force on the magnetic poles tends to give positive rotation velocities $v$ in case $(a)$ negative velocities in case $(b)$ and zero velocity in case $(c)$ (since $v=0$ on the outer surface, where the force is applied). 
geneities in the applied field or in the fluid itself (local variations of the concentration, the viscosity, etc...). As Moskowitz and Rosensweig pointed out in reference [1], these rotations are potentially interesting for technical applications and the problem of driving a fluid by a rotating magnetic field has come to present interest [1], [2], [3], [4] with the recent availability of ferrofluids.

Two sets of observations have been made on a chemically prepared cobalt ferrofluid and on a magnetic suspension of large $\mathrm{CrO}_{2}$ grains. Both sets are in total agreement. But the " discrete " suspension of grains is more suited than the "continuous » ferrofluid for observation and description of the local motions and we will refer essentially to that suspension only in the following.

2. Experimental techniques. - 2.1 THE FLUID. 2.1.1 $\mathrm{CrO}_{2}$ suspension. - The fluid consists of small particles of $\mathrm{CrO}_{2}$ in suspension in an isotropic carrier liquid : water, toluene, alcohol, for example. The particles are acicular in shape, about $100 \AA$ wide and a few $1000 \AA$ long. We used fluids of various concentrations ranging from 0.2 to $5 \%$ in weight. First, we apply momentarily a static field of about 200 Oe which leads to particle agglomeration. The fluid becomes a suspension of irregularly shaped grains about $100 \mu$ in diameter. In the absence of motion, these suspensions would precipitate with time (the particules not being coated with any surfactant molecules) but under rotating fields the grains are permanently dispersed by the strong internal agitation. A typical rotating suspension is shown on plate $I$.

2.1.2 Cobalt ferrofluid. - The ferrofluid has been prepared in our laboratory by Liebert and Strzelecki following the method described in references [5] and [6]. It consists of roughly spheroidal cobalt grains, $80 \AA$ in size. The grains are coated with a terpolymer mixture and suspended in a toluene-carrier. Typical magnetization are $80 \mathrm{G} / \mathrm{cm}^{3}$. The dispersion is stable in time and in fields up to at least $10 \mathrm{kG}$.

2.2 The APparatus. - The fluid is placed in a small horizontal cylindrical container about $1 \mathrm{~cm}$ in diameter and a few millimeters high. A rotating magnetic field is produced in the plane of the container by two orthogonal pairs of Helmholtz coils, driven $90^{\circ}$ out of phase by a common RF signal. The fields of magnitude 0 to $100 \mathrm{Oe}$ and of frequency 5 to $500 \mathrm{~Hz}$, are spatially uniform within $1 \%$ over an area of about $4 \mathrm{~cm}^{2}$. In the experiments to be described below, the frequency of the rotating field was $50 \mathrm{~Hz}$ (unless otherwise mentioned). Furthermore, a third pair of coils can be used to produce a vertical, static and uniform magnetic field of about 100 Oe. Some other small coils (about $1 \mathrm{~mm}$ in diameter) can be used to create a locally non-uniform static field. The whole set-up is mounted under an optical microscope for observations from $10 \times$ to $1000 \times$. Direct visual obser-

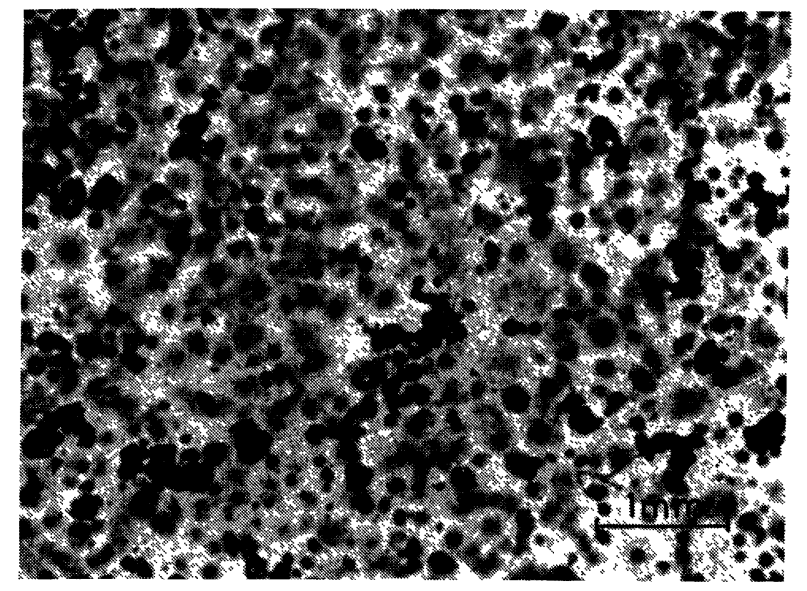

(a)

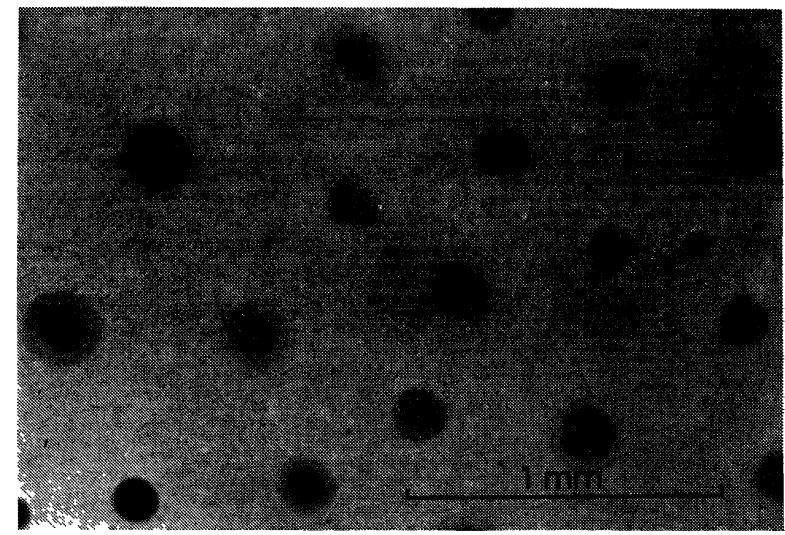

(b)

Plate I

a) Typical micrograph of a rotating suspension.

b) For lower concentrations, vortices extend vertically throughout the sample.

vation, photographs and moving pictures were used. The grains are driven synchronously by a 100 Oe rotating field till a maximum frequency of about 300 or $400 \mathrm{~Hz}$. At higher frequencies, they no longer lock on the field but still have a very slow rotation. In what follows, we are always in the synchronous rotation regime for the grains $\left(^{1}\right)$.

3. Results. - The bulk rotation $R$ when present is said to be positive if it is in the same direction as the rotating field, negative otherwise. A bulk rotation means that a steady rotation takes place with an average radius-dependent angular velocity. But a particular volume element of the suspension follows a circular path only over a fraction of turn, say a fourth or a half, and then diffuses in an erratic way. The velocity profile has not been determined precisely. Unless otherwise mentioned, $R$ is intended for the radius where the linear velocity is maximum.

(1) But note that the rotation of the liquid, when it occurs, is much slower and never synchronous. 
CASE I. - Uniform concentration. Uniform field. Each grain drives the adjacent liquid into rotation. Local disturbances and convection-like flows are observed, but are essentially random and no net bulk rotation is observed (less than $0.1 \mathrm{rpm}$ for $H_{\mathrm{r}}=100 \mathrm{Oe}$ and $100 \mathrm{~Hz}$ ). This result is in apparent contradiction with the data of reference [1]. However, we have found a number of secondary effects which can explain this apparent discrepancy.

i) With highly concentrated solutions, the interparticle attractive forces (internal cohesion) tend to pull the grains slightly towards the center. A very narrow ring of lower concentration appears at the outside, and there is a marked positive rotation for these few outside layers $(R \sim 1.5 \mathrm{~Hz}$, maximum linear velocity observed $: 5.3 \mathrm{~cm} / \mathrm{s}$ ) rapidly decreasing towards the centre.

ii) In presence of a static vertical magnetic field, the grains tend to flip up vertically and are mutually repelled forming a stable lattice. At low concentrations, the grains can thus be driven at much higher frequencies and perform a precession on a narrow cone. The mutually repulsive forces squeeze the outer layer against the boundary of the container. The outer layers show a slight negative rotation, limited to the first few layers.

CASE II. - Strongly non-uniform magnetization and fields. - These two effects are very hard to separate. A non-uniform field tends to concentrate the grains in the high field region. Conversely, non-uniform concentration would distort the field configuration locally. However, several clear cut situations were observed :

i) A small coil located at the center of the sample creates a non-uniform static field, which pulls the solution radially inwards (Fig. 2). There is a marked positive bulk rotation; the steeper the concentration gradient the higher the rotation rate is.

ii) The container is filled with two non-miscible fluids, one containing the grains, the other being nonmagnetic. The radial distribution of $M$ is a step function as on figure 1 . If the grains are in the inner fluid ring, we find that the rotation is positive, negative in the opposite case.

iii) A meniscus is formed in an open container (Fig. 2). A concave meniscus (corresponding to an excess of grains on the outside) shows negative rotation. A convex one shows positive rotation $\left({ }^{2}\right)$.

(2) Generally speaking, a negative rotation would be more stable than a positive one. For instance, in paragraph 3, case I, i), when there is a very strong initial positive rotation, the centrifugal force tends to accumulate the grains at the outside : this leads then to a negative rotation for the central part. This rotation stabilizes itself because the centrifugal force maintains the same sign for the concentration gradient which caused it. This could perhaps be the explanation for the inversion from forward to reverse rotation reported in reference [4].
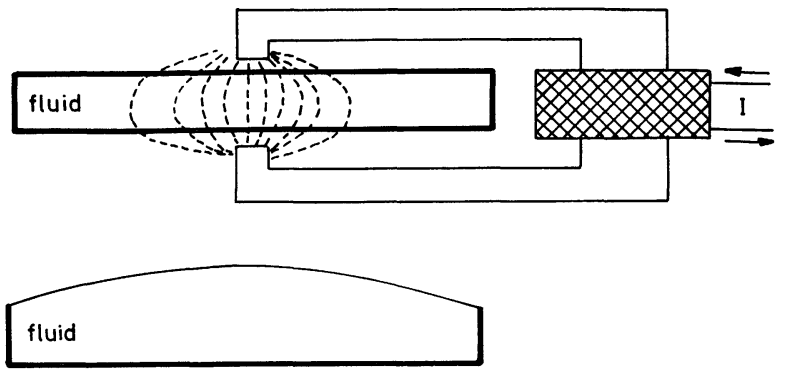

$R>0$

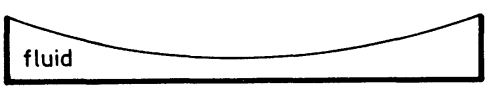

$\mathrm{R}<\mathrm{O}$

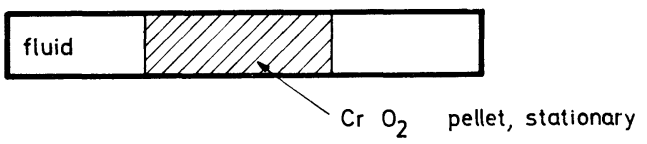

FIG. 2. - Vertical cross section of some experimental situations discussed in paragraph 3 . The applied homogeneous rotating field $\mathbf{H}$ rotates in a horizontal plane. $R$ is the bulk rotation.

iv) A solid pellet made by agglomeration of the same $\mathrm{CrO}_{2}$ powder in an adhesive is placed at the center of the container (Fig. 2). It perturbs concentration, local static field and rotating field. Without applied vertical field, the grains are attracted by the central pellet. There is a strong positive rotation. With an applied vertical field, they are pushed out by the pellet (which is also vertically magnetized in the same sense) and the bulk rotation is inverted. If now a reversed vertical field lower than the coercive force of the magnetized pellet is applied again the grains are attracted by the pellet and a very strong positive bulk rotation follows.

CASE III. - Non-uniform viscosity. - Starting with a uniform water-base solution showing no bulk rotation, heat is generated at the center by contact with a hot copper rod and a temperature gradient of $100 \% \mathrm{~cm}$ is maintained: this creates a central region with reduced viscosity. We observe a negative bulk rotation of about $0.1 \mathrm{~Hz}\left({ }^{3}\right)$.

4. Interpretation. - 4.1 MAGNETIC DESCRIPTION : MAGNETIC POLES. - Each volume element in the fluid can be assigned a magnetization $\mathbf{M}$, of a given magnitude, related to the concentration, and showing a given lag angle $\alpha$ with respect to the applied field. The lag angle characterizes the viscous drag of this volume element. The larger the friction, the greater the lag angle, so that the resulting torque $\mathbf{C}=\mathbf{M} \times \mathbf{H}$

(3) We have also observed these effects of a viscosity gradient, without any temperature gradient, by inducing some aggregation between the grains through a radially dependent magnetic force (leading to a more viscous phase). 
will always drive the volume element at a frequency synchronous to the field. The knowledge of $|\mathbf{M}|$ and $\alpha$ gives the distribution of magnetic poles in the suspension, this allows the calculation of the external forces which may promote a particular rotation. Of course, the magnetic poles description is only a mathematical device, but it is a convenient tool to visualize the qualitative behavior of the suspension. More elaborate formulation of the stress of a deformable magnetic material and its equivalence to the magnetic poles description can be found in reference [7]. There will be bulk rotation when magnetic poles are present in the liquid, i. e. whenever $|\mathbf{M}|$ or $\alpha$, or both are non-uniform throughout the sample, as, can be illustrated in the following example :

Consider a sample where there are two concentric areas of different viscosities so that the lag angle will be greater in the center say (Fig. 3). Magnetic poles appear at the separation between the two domains. In this particular case, the bulk rotation will be positive in the vicinity of the interface. With this same magnetic poles model, we could explain any of the results of paragraph 3 .

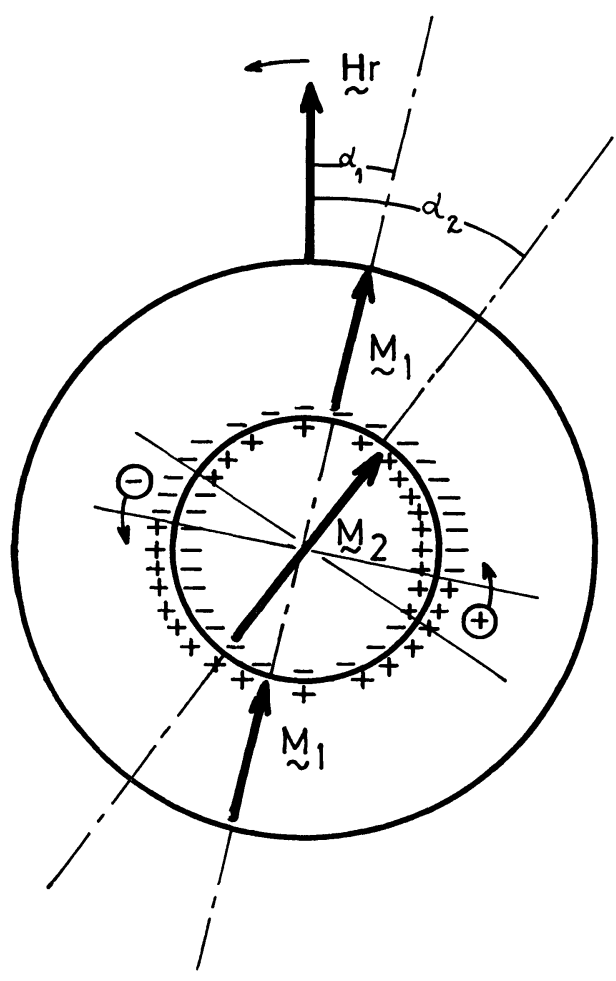

$\left.{ }^{F}\right)_{G}$. 3. - The variation of the lag angle throughout the sample, induces magnetic poles inside the liquid which lead to a rotation (positive in the particular case shown).

The advantage of the above model is that it automatically takes into account the nature of the carrying fluid or the discreteness of the grains distribution. It can be carried to the limit of a fluid continuum. Therefore the objection that could be raised about our fluid not being a true homogeneous ferrofluid vanishes.
Qualitatively, the same behavior will be expected from any other colloidal magnetic fluid. In fact, we performed observations on our ferrofluid and all conclusions reached in paragraph 3 still hold.

4.2 MiCROSCOPIC ASPECTS. - The bulk behavior of a suspension of magnetic particles can be calculated from the study of the microscopic motions of the grains [2], [8], [9]. These calculations have been carried out explicitly in the case of uniform suspensions. In the more complex situation of our experiments for which we have no accurate quantitative measurements, it might still be instructive to observe the motion of individual grains and the motion of the fluid around them. When a grain is forced into rotation by the rotating field $H_{\mathrm{r}}$, it creates a circular backflow. The bulk fluid velocity is the sum of all these individual backflows. When the fluid is macroscopically uniform, there is an exact compensation, the sum averages to zero. But near one grain $G$ the backflow due to $\mathrm{G}$ dominates and remains clearly visible.

Departures from the macroscopic averages are particularly important near a wall: in the sheet between the last grains and the wall, we constantly observe a positive rotation, which is clearly dominated by the backflow of the last grains. Note that this rotation is also in agreement with the model of figure 1 , the magnetic poles being located on the last grains and not strictly on the walls due to the finite size of the grains (cf. $\S 3$, case I, i).

Another effect occurs, if the friction is not the same on both sides of the grains or of the associated vortex. A net force appears. This is a very general case leading to bulk rotation, whenever there is a spatial variation of any parameter (concentration, viscosity, fields, ...). If the friction is increasing towards the center, the lag angle increases too and we have positive rotation in agreement with figure 3 .

Thus, friction of a grain against a boundary, an interface or another array of grains, leads to a rolling motion of the grain. This effect is somewhat balanced by the wall effect. The wall effect would dominate at higher frequencies, when the flow is appreciable, the rolling effect at lower frequencies, when the grain is strongly applied against a surface by some other force (e. g. the magnetic repulsion of $\S 3$, case $I$, ii).

Also, intergrain cohesive forces due to near neighbor interactions whether hydrodynamic or magnetic in nature, can lead to a spatially dependent friction. An hydrodynamic example is shown in figure 4 . The relative velocities of the vortex (1) with respect to the surroundings are $(V)$ at $\mathrm{A}$, and $(2 V)$ at $\mathrm{B}$. The resulting frictions are different and vortex (1) drifts along with a velocity $v_{1}$. This modifies the velocity balance for vortex (2): $\left(2 V-v_{1}\right)$ at $\mathrm{B}$ and $(2 V)$ at $\mathrm{C}$, so that vortex (2) now drifts with velocity $v_{2}$. A second order effect also appears. The velocity competition for (1) has become $\left(2 V+v_{1}\right)$ at $\mathrm{A}$ and $\left(2 V-v_{1}+v_{2}\right)$ at $\mathrm{B}$, so that (1) drifts with a velocity $\left(v_{1}+\delta v_{1}\right)$. Likewise 


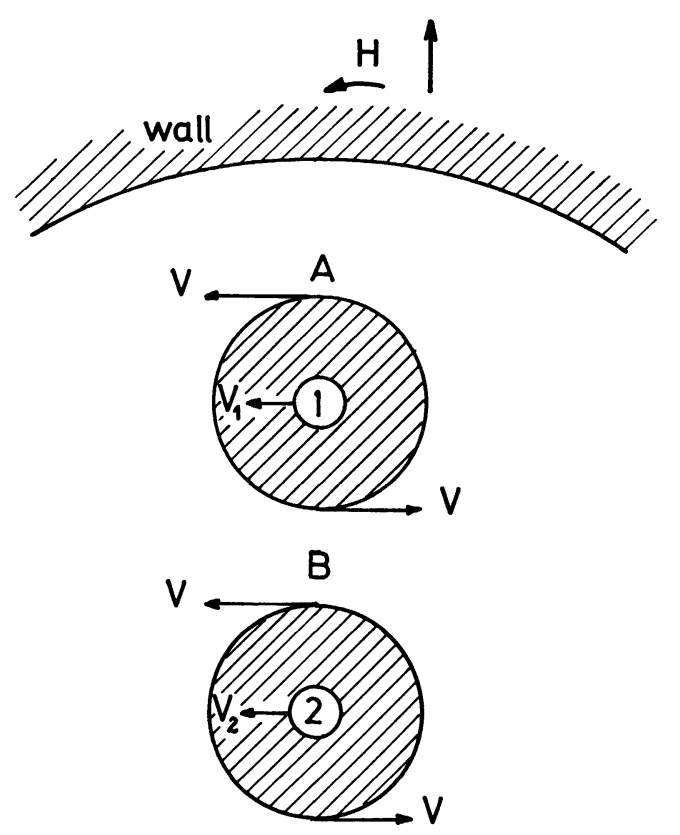

C

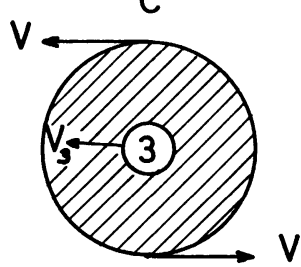

Fig. 4. - The spatial dependent friction between the grains (larger at $B$ than at A, etc..., see text) induces a bulk positive rotation in the vicinity of the wall $\left(v_{1}>v_{2}>v_{3} \cdots\right)$.

(2) drifts with velocity $\left(v_{2}+\delta v_{2}\right)$, etc... This cascading process has to be carried to higher orders, and (1) will drift with velocity $\left(v_{1}+\delta v_{1}+\delta^{\prime} v_{1}+\cdots\right)$,
(2) with velocity $\left(v_{2}+\delta v_{2}+\delta^{\prime} v_{2}+\cdots\right)$, etc... The final result is a bulk positive rotation, decreasing in magnitude from the wall to the center.

Any accidental inhomogeneity (effects of the walls, field concentration or temperature gradients, etc...) could have been responsible for the circulations observed in reference [1].

5. Conclusions. - Some of the preceding mechanisms are very localized and are generally confined to the boundaries or to some peculiar layers. They are due to the heterogeneous character of the suspension. Friction, non compensation of the individual backflows or viscosity effects, on the other hand, can arise anywhere in the fluid and can be cooperative. They are still possible in the continuum limit. They are more efficient to induce bulk rotation.

Our observations are still very qualitative at the present stage. However, they do prove certain points unambiguously :

- In a uniformly magnetized fluid, a rotating field induces no bulk circulation.

- The sign of the rotations observed in inhomogeneous situations may vary but is always correctly given by simple considerations based on magnetic pole densities.

- Strong inhomogeneities and rotations can be induced and modulated in time either by auxiliary fields or by heat sources.

Acknowledgments. - We wish to thank P. G. de Gennes for his interest and for helpful discussions.

\section{References}

[1] Moskowitz R. and Rosensweig R. E., Appl. Phys. Lett. 11 (1967) 301.

[2] Jenkins J. T., J. Physique 32 (1971), 931.

[3] Laithwaite E. R., Electrical Rev. 182 (1968) 130.

[4] Brown R. and Horsnell T. S., Electrical Rev. (1969) 235.

Brown R., Birch T. S., Mc Cormick M., Proc. EM 70, Conf. Univ. Dundee (England).
[5] Thomas J. R., J. Appl. Phys. 37 (1966) 2914.

[6] Hess P. M. and Parker P. H. Jr., J. Appl. Polymer Science 10 (1966) 1915.

[7] Brown W. F. Jr., Springer tracts in Natural Philosophy, Vol. 9, p. 50 and 59 N. Y. Springer 1966.

[8] BAtchelor G. K., J. Fluid Mech. 41 (1970) 545-70.

[9] LeAl L. G., J. Fluid Mech. 46 (1971) 395-416. 